\title{
QUIMIODECTOMA EM CÃO - RELATO DE CASO
}

Lígia Fernandes Gundim", Thaís de Almeida Moreira ${ }^{2}$, Rafael Rocha dos Souza ${ }^{2}$, Márcio de Barros Bandarra ${ }^{3}$, Alessandra Aparecida Medeiros ${ }^{3}$

1- Mestranda no Programa de Ciências Veterinárias da Universidade Federal de Uberlândia, Uberlândia-MG. E-mail: ligia_fg@hotmail.com.

2- Residente em Patologia Animal, Universidade Federal de Uberlândia, Uberlândia - MG.

3- Professor doutor em Patologia Animal, Universidade Federal de Uberlândia, Uberlândia - MG.

Recebido em: 08/09/2015 - Aprovado em: 14/11/2015 - Publicado em: 01/12/2015 DOI: http://dx.doi.org/10.18677/Enciclopedia_Biosfera_2015_218

\begin{abstract}
RESUMO
Os quimiodectomas são neoplasias incomuns que se originam dos quimiorreceptores situados, principalmente, no corpo aórtico e corpo carotídeo. Este trabalho teve como objetivo relatar o caso de um cão, sete anos, Fila Brasileiro, que foi encaminhado ao setor de Patologia Animal da Universidade Federal de Uberlândia para realização de necropsia. À necropsia observou-se massa em base da aorta, medindo aproximadamente $6 \times 4 \mathrm{~cm}$ de diâmetro, de consistência firme, coloração avermelhada, ao corte áreas claras intercaladas a áreas de coloração vermelho escuro. Microscopicamente observou-se células exibindo moderado pleomorfismo, arredondadas a poliédricas, eosinofílicas, citoplasma finamente granular, núcleo central e esférico, demonstrando ainda estroma fibrovascular rico em colágeno de entremeio. A análise histopatológica foi compatível com quimiodectoma.
\end{abstract}

PALAVRAS- CHAVE: coração, caninos, neoplasia, quimiorrectores.

\section{CHEMODECTOMA IN DOG - CASE REPORT}

\begin{abstract}
The chemodectomas are rare neoplasms derived from chemoreceptors mostly located in the aortic body and carotid body. The objective was report the case of a dog, seven age, Fila Brasileiro, which was referred to the Animal Pathology Department, Federal University of Uberlândia for necropsy. At necropsy there was a mass base of the aorta, measuring approximately $6 \times 4 \mathrm{~cm}$ in diameter, firm consistency, reddish color, that cut clear areas interspersed with dark red areas. Microscopically observed pleomorphic, rounded to polyhedral eosinophilic cells, finely granular cytoplasm, central and spherical nuclei, still demonstrating fibrovascular stroma rich in collagen inset. Histopathology was compatible with chemodectoma.
\end{abstract}

KEYWORDS: cancer, heart, quimiorrectores, canines. 


\section{INTRODUÇÃO}

Os quimiodectomas ou paragangliomas são neoplasias de quimiorreceptores que ocorrem na base do coração, e ao contrário das neoplasias primárias do coração, que são incomuns, podem ocorrer em cães e raramente em gatos e bovinos (CAPEN, 2002). Os quimiorreceptores são estruturas responsáveis pela conservação da homeostase do sistema cardiorrespiratório, detectando variações do pH do sangue, pressão de oxigênio (PO2) e de dióxido de carbono (PCO2). Podem atuar também como barorreceptores, causando aumento ou diminuição da freqüência cardíaca, pressão arterial, tônus vasomotor, atividade do córtex cerebral e liberação de adrenalina (EHRHART et al., 2002). Estes tumores raramente têm comportamento maligno malignos, entretanto podem apresentar metástases em pulmões e fígado (ARAÚJO et al. 2011).

Como diagnóstico diferencial, há que se atentar a hemangiossarcomas, carcinomas de tireoide ectópicos (MESQUITA et al., 2012), linfomas cardíacos (ANAl, et al., 2013) e mesoteliomas (Ware, 2010) uma vez que ocorrem frequentemente em cães em locais comuns ao quimiodectoma e apesar de apresentaram origem extracardíaca podem causar obstrução e falha cardíaca devido à sua localização.

Cães idosos e de raças braquiocefálicas, como Boxer, Bulldog e Boston Terrier, apresentam maior incidência devido à conformação anatômica de narinas curtas, estenóticas e palato mole alongado (CAPEN, 2002). Usualmente estes tumores se apresentam como massas únicas ou múltiplas dentro do saco pericárdico, perto da base do coração, variando entre 0,5 a $12,5 \mathrm{~cm}$, superfície lisa e ao corte são brancacentos, intercalados com áreas vermelho amarronzadas (CAPEN, 1991)

Microscopicamente, as células são bem diferenciadas, cubóides, núcleos esféricos pontilhados e citoplasmas vacuolizados. As células usualmente estão arranjadas em pequenos aglomerados e separadas por um estroma fibroso (CHIQUITO et al., 1998 e CAPEN, 2002).

O presente estudo teve como objetivo relatar o caso de um canino atendido no Hospital Veterinário da Universidade Federal de Uberlândia diagnosticado com quimiodectoma.

\section{RELATO DE CASO}

Um cão, macho, sete anos de idade, Fila brasileiro, foi encaminhado ao setor de Patologia animal, do Hospital Veterinário da Universidade Federal de Uberlândia para realização de necropsia. Conforme dados do histórico, o animal veio à óbito, sendo relatado apenas sintomatologia compatível com cinomose.

\section{RESULTADOS E DISCUSSÃO}

$\mathrm{Na}$ necropsia observou-se massa na base da aorta medindo aproximadamente $6 \times 4 \mathrm{~cm}$ de diâmetro, de consistência firme, coloração avermelhada, ao corte áreas claras intercaladas a áreas de coloração vermelho escuro (Figura 1). Fragmentos da massa neoplásica foram coletados e fixados em formol $10 \%$ por 48 horas e processados rotineiramente para posterior análise histopatológica. 


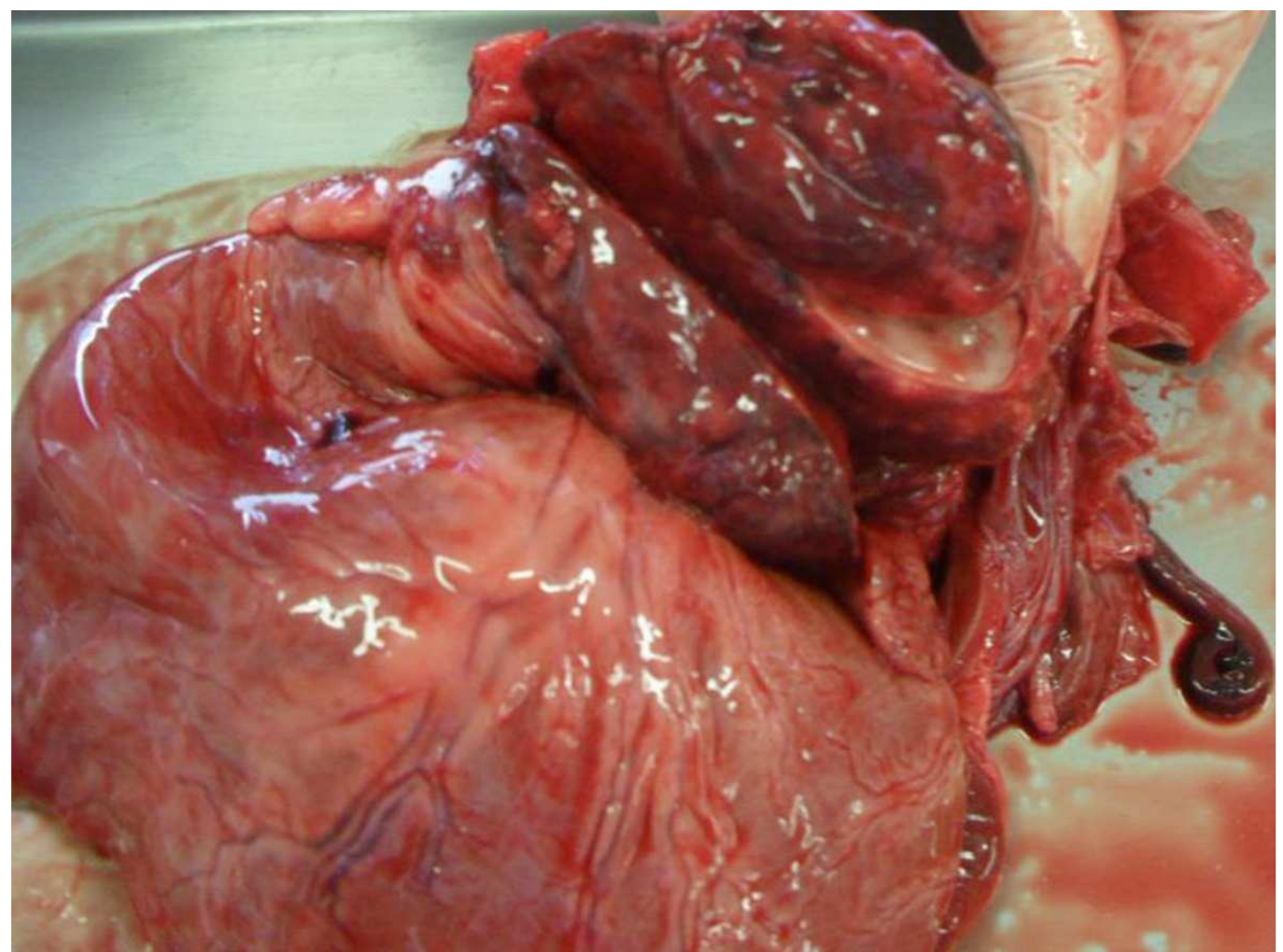

FIGURA 1 - Cão, Fila Brasileiro, sete anos de idade. Massa firme, avermelhada, localizada na base da aorta.

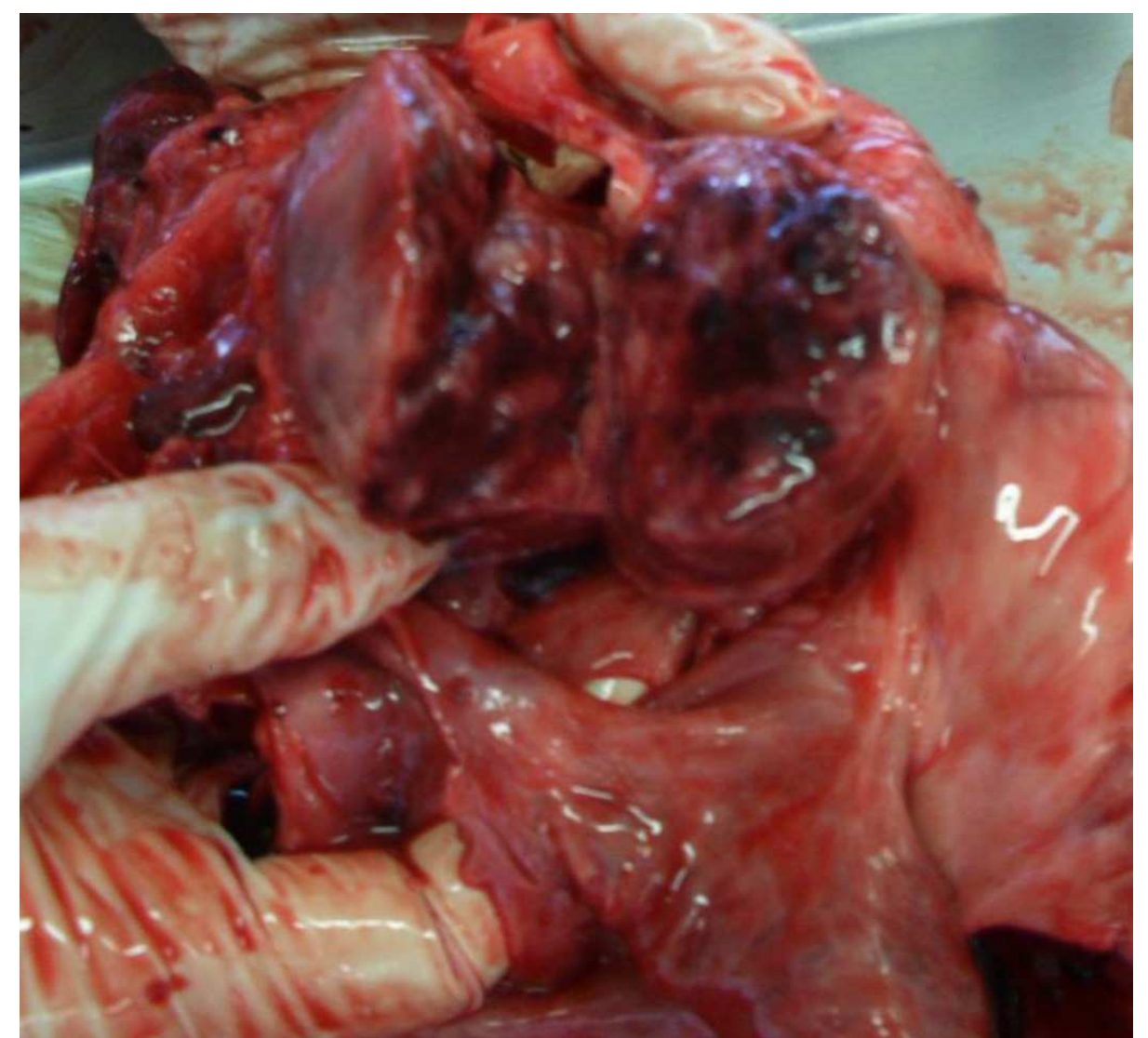

FIGURA 2 - Cão, Fila Brasileiro, sete anos de idade. Aspecto da massa ao corte, demonstrando áreas claras intercaladas a áreas vermelho escuro. 


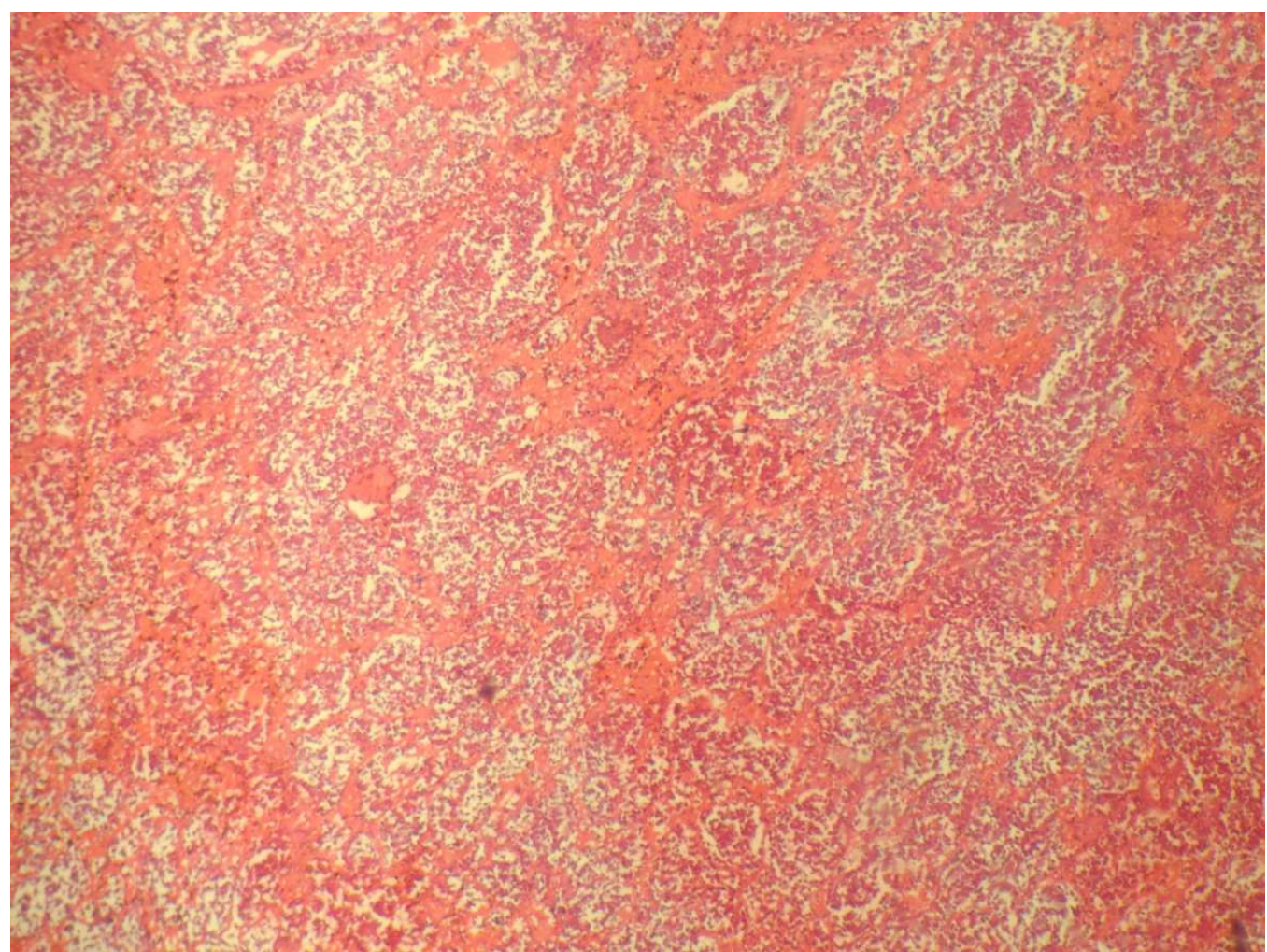

FIGURA 3- Cão, Fila Brasileiro, sete anos de idade. Observar abundante estroma fibrovascular rico em colágeno de entremeio. Aumento de 40x.

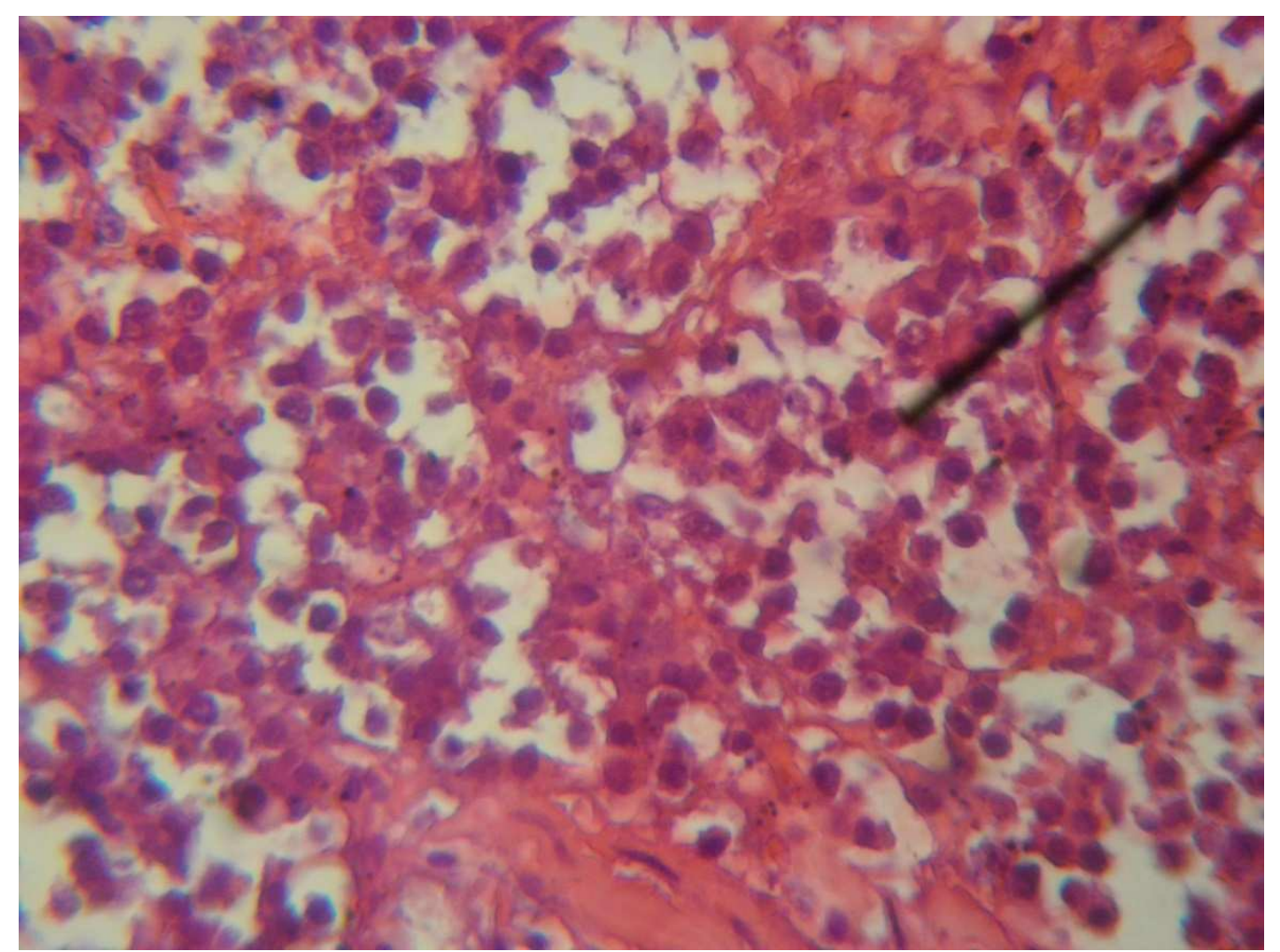

FIGURA 4 - Cão, Fila Brasileiro, sete anos de idade. Notar células pleomórficas, eosinofílicas, de núcleo central e esférico. Objetiva de 40x. Coloração H.E. 
Tumores originários dos tecidos quimiorreceptores apresentam baixa incidência dentre as diversas neoplasias caninas (MOURA et al., 2006). Em estudo realizado na região do Paraná avaliando um período de 21 anos, registrou-se apenas cinco casos de quimiodectomas em cães (CHIQUITO et al., 1998). O primeiro relato de quimiodectoma em cão na região de Uberlândia foi realizado há 15 anos (FERREIRA et al., 2000), não sendo encontrados até o momento outras descrições de ocorrência deste tumor na mesma cidade, demostrando assim a rara ocorrência da neoplasia. Segundo CAPEN (2002), cães de raças braquiocefálicas, como Bulldog, Boston Terrier e Boxer apresentam maior incidência da doença. Entretanto, neste estudo o animal acometido era da raça Fila Brasileiro.

Síncope e cansaço fácil são sinais que podem ser observados em cães portadores deste tumor, podendo ser identificados fibrilação atrial e extra-sístoles ventriculares isoladas no exame eletrocardiográfico (CAVALCANTI et al., 2006). Outros sinais clínicos inespecíficos como tosse seca e edema de membros também foram relatados na literatura (CARVALHO et al., 2011). Os exames: radiográfico, ultra-sonográfico e ecocardiográfico podem demonstrar hepatomegalia, efusão peritoneal, pleural e pericárdica, bem como a visualização da massa cardíaca (ALMEIDA et al.,2002). Neste caso, não foi possível observar a sintomatologia clínica uma vez que o animal foi encaminhado somente para realização de necropsia, não acompanhado de histórico.

Os achados macroscópicos são semelhantes aos descritos na literatura, sendo, como neste caso, a localização da massa na base da aorta (ZIMPEL, et al., 2013; SALOMÃO et al., 2012), entretanto não foi observada hipertrofia excêntrica do ventrículo direito como relatado por MESQUITA et al., (2012).

O pulmão é o local onde a ocorrência de metástase deste tumor é mais relatada (SALOMÃO et al., 2012;), porém pode ocorrer também em linfonodos bronquiais e mediastínicos, fígado, pâncreas e rins (CAPEN, 1991). Não obstante, neste caso não foi observada a presença de metástase.

As células tumorais observadas apresentavam-se algumas com formato arredondado, outras poliédricas, eosinofílicas, finamente granular. Estes achados corroboram com o observado por ZIMPEL et al. (2013), os quais identificaram ainda, a presença de células gigantes mononucleadas e fendas de colesterol. Visualizou-se ainda áreas multifocais de necrose e hemorragia, assim como descrito por ABREU et al., (2014)

Conforme CAPEN (1991), nódulos únicos ou múltiplos no saco pericárdico, próximos à base do coração, sugerem ter origem no corpo aórtico, sendo este provavelmente o local de origem do tumor observado neste caso, tendo em vista os achados evidenciados.

\section{CONCLUSÃO}

O quimiodectoma é uma neoplasia rara, apresentando desta forma poucos trabalhos na literatura que o abordem. O conhecimento deste tipo de tumor, bem como da sintomatologia associada, torna-se importante na clínica veterinária, principalmente na diferenciação de outras patologias. O paciente pode apresentar-se assintomático, sendo o tumor encontrado em exames radiográficos de rotina ou como achados de necropsia, permitindo o diagnóstico através da análise histopatológica. Neste caso, os achados levam a crer que o tumor teve origem na base da aorta. 


\section{REFERÊNCIAS}

ANAI, L. A.; JARK, P.C.; TERRA, E.M.; GAVA, F.N.; MARINHO, F.A.; CALAZANS, S.G.; COSTA, M.T.; SANTANA, A.E. Linfoma Cardíaco primário em cão. Semina: Ciências Agrárias, p. 2375-2379, 2013. Disponível em: <http://repositorio.unesp.br/handle/11449/112480>. Doi: 10.5433/1679$0359.2013 v 34 n 5 p 2375$

ARAÚJO, M.M.; CARANDINA, L.S.; PRADA, T.C.; COELHO, V.S.; ZANCO, N.A.; COUTINHO, A.S. Quimiodectoma em um cão. Revista de Educação Continuada em Medicina Veterinária e Zootecnia, v.0, n.2, p. 46-47, 2011. Disponível em: < http://revistas.bvs-vet.org.br/recmvz/article/view/444/415>

ALMEIDA, M.R.P.; STERMAN, F.A.; SARRAF, A.P.; URTADO, S.R.; FELIZZOLA, C.R. Quimiodectoma no átrio direito em beagle - relato de caso. In: Congresso Brasileiro de Medicina Veterinária (CONBRAVET), 29, 2002, Anais... Gramado:SBMV/SOVERGS. p.1282. Disponível em: <http://www.sovergs.com.br/site/conbravet2002/1282.htm>.

Data de acesso:09 Jun 14.

CAPEN, C.C. Patologia de los Animales Domésticos. In: JUBB, K.V.F.;KENNEDY, P.C.; PALMER, N. Montevideo: Agropecuária Hemisfério Sur, 1991. cap.3. Vol.3. Pathology of Domestic Animals. p. 331- 333.

CAPEN, C.C. Tumors of the Endocrine Glands. In: MEUTEN, D. Tumors in domestic animals, 4 ed. Ames: lowa State, 2002. p. 601-695.

Carvalho, T. F.; Coelho, H. E.; Machado, F. M. E.; Alberto, H.; Locce, C. C.; Silva, K. D. C.; Magalhães, A. O. C. Ocorrência de quimiodectoma em tronco carotídeo de cão. Pubvet, Londrina, v. 5, n. 31, 2011. Disponível em: < http://www.pubvet.com.br/uploads/4094ca5cfb6c7d64f06939a40cd89c5f.pdf>

CAVALCANTI, G.A.O; MUZZI, R.A.L.; BEZERRA JÚNIOR, P.S.; NOGUEIRA, R.B.; VARASCHIN, M.S. Fibrilação atrial em cão associada ao quimiodectoma infiltrativo atrial: relato de caso. Arquivo Brasileiro de Medicina Veterinária e Zootecnia, v.58, n.6, p.1043-1047, 2006. Disponível em: < http://www.scielo.br/pdf/abmvz/v58n6/11.pdf>

CHIQUITO, M.; WERNER, P.R.; PACHALY, J.R. Ocorrência de quimiodectomas em animais no primeiro planalto do Paraná, Brasil. Arquivo de Ciências Veterinárias e Zoologia da Unipar v.1, n.1, p. 67-70. 1998. Disponível em: < http://revistas.unipar.br/veterinaria/article/view/633/550>

EHRHART N, EHRHART EJ, WILLIS J, SISSON D, CONSTABLE P, GREENFIELD C, MANFRA-MARETTA S, HINTERMEISTER J. Analysis of factors affecting survival in dogs with aortic body tumors. Veterinary Surgery, v.31, n.1, p.44-48, 2002.

Disponível em: < http://onlinelibrary.wiley.com/doi/10.1053/jvet.2002.29989/pdf>

FERREIRA, F.A; FREIRE, M.G.M; COELHO, H.E.; FERREIRA, C.G; MANZAN, R.M. Quimiodectoma de corpo carotídico em um cão. Veterinária Notícias. v.6, n.1, p 107-111. 2000. 
MESQUITA, L.P., ABREU, C.C., NOGUEIRA, C.I., WOUTERS, A.T.B., WOUTERS, F. BEZERRA-JUNIOR, P.S., MUZZI, R.A.L., VARASCHIN, M.S. Prevalência e aspectos anatomopatológicos das neoplasias primárias do coração, de tecidos da base do coração e metastáticas, em cães do Sul de Minas Gerais (1994-2009). Pesquisa Veterinária Brasileira, v. 32, n.11, p. 1155-1163, 2012. Disponível em: < http://www.scielo.br/pdf/pvb/v32n11/a14v32n11.pdf>

MOURA, V.M.B.D; GOIOZO, P.F.I; THOMÉ, H.E; CALDEIRA, C.P; BANDARRA, E.P. Quimiodectoma como causa de morte súbita em cão - relato de caso. Veterinária Notícias., Uberlândia, v. 12, n. 1, p. 95-99, 2006. Disponível em: < https://scholar.google.com.br/scholar?hl=pt-

$\mathrm{BR} \& \mathrm{q}=$ Quimiodectoma+como+causa+de+morte+s\%C3\%BAbita+em+c\%C3\%A3o+\% $E 2 \% 80 \% 93+$ relato+de+caso\&btnG $=\& \mid r=>$

SALOMÃO, M.C.; MATTOS, A.S.; LUCENA, A.R.; LEITE, J.S.; MELLO, M.F.V.; FERREIRA, A.M.R. Tumor de arco aórtico em cão (Canis familiaris)-relato de caso. Veterinária e Zootecnia, v. 19, n. 1-S. 1, p. 107-109, 2012. Disponível em: <http://www.fmvz.unesp.br/rvz/index.php/rvz/article/view/249/206>

Ware, W.A. Doenças do sistema cardiovascular In: Couto, C.G., Nelson, W.R. Medicina interna de pequenos animais. 4.ed. Missouri: Mosby, 2010

ZIMPEL, A.V.; AGUIAR, P.F.; ROSSATO, C.K. Quemodectoma aórtico em canino relato de caso. 2013. In: Seminário Interstitucional de Ensino, Pesquisa e Extensão, 23, 2013, Anais... Cruz Alta: Unicruz. Disponível em: < http://www.unicruz.edu.br/seminario/anais/2013/CCS/MEDICINA\%20VETERINARIA/ C.Oral/QUEMODECTOMA\%20A\%D3RTICO\%20EM\%20CANINO\%20\%96\%20REL ATO\%20DE\%20CASO.> 